\title{
Synchronous tumours detected during cancer patient staging: prevalence and patterns of occurrence in multidetector computed tomography
}

\author{
Antonio Corvino ${ }^{1 A, B, C, D, E, F}$, Sergio Venanzio Setola ${ }^{2 B}$, Fabio Sandomenico ${ }^{2 B}$, Fabio Corvino ${ }^{3 A, C, F}$, \\ Orlando Catalano $0^{2 A, B, E, F}$ \\ ${ }^{1}$ Motor Science and Wellness Department, University of Naples, Naples, Italy \\ ${ }^{2}$ Radiology Department, National Cancer Institute Pascale Foundation, Naples, Italy \\ ${ }^{3}$ Vascular and Interventional Radiology Department, Cardarelli Hospital, Naples, Italy
}

\section{Abstract}

Purpose: The incidental detection of one or more additional primary tumours during computed tomography (CT) staging of a patient with known malignancy is rare but possible. This occurrence should be considered by the radiologist when a new lesion is detected, especially if the lesion location is atypical for metastases. The purpose of this report was to document the usefulness of total body CT scan to detect synchronous primary malignancies in cancer patients undergoing a staging workup.

Material and methods: This was done by reviewing the staging CT studies of the adult patients with a newly diagnosed cancer evaluated during a five-year period in a single cancer institute in order to identify any possible correlation, establishing which tumours are more frequently combined with a second tumour and which second tumours are more commonly present.

Results: Among the patients with a second tumour, the most frequent first primary tumours were melanoma (eight patients, $17.8 \%$ ), lymphoma (seven patients, $15.6 \%$ ), and prostate carcinoma (seven patients, $15.6 \%$ ). The most frequent incidentally detected second tumours were hepatocellular carcinoma (nine patients, $20 \%$ of 45 incidental tumours), renal carcinoma (eight patients, 17.8\%), lung carcinoma (seven patients, 15.6\%), and bladder carcinoma (four patients, $8.9 \%$ ). One patient had three primary tumours synchronously.

Conclusions: We believe that the radiologist's knowledge of the prevalence and pattern of occurrence of these multiple primary malignancies represents added diagnostic value.

Key words: diagnostic imaging, computed tomography (CT), multiple primary malignancies (MPMs), synchronous tumours, multiphasic CT protocol.

\section{Introduction}

Multiple primary malignancies (MPMs), defined as two or more malignant primary tumours arising in the same patient, are an uncommon but well recognised medical evidence, especially for clinicians and oncologists $[1,2]$.
MPMs present the following clinical and histological characteristics: (1) malignant tumours based on histopathologic criteria, (2) topographic distinction without connection (skip lesions), and (3) ruling out that the second tumour is not a metastasis of the first. This avoids misclassification of multifocal or multicentric tumours or

Correspondence address:

Dr. Antonio Corvino, Motor Science and Wellness Department, University of Naples “Parthenope", via F. Acton 38, I-80133 Naples, Italy, e-mail: an.cor@hotmail.it Authors' contribution:

A Study design · B Data collection · C Statistical analysis · D Data interpretation · E Manuscript preparation · F Literature search · G Funds collection 
metastases as multiple primaries. A cancer is classified as index cancer if there has been no prior record of invasive cancer. In addition, a temporal classification describes MPMs as synchronous or metachronous, depending on whether diagnosis of the second tumour was made within or after six months of the first ("index tumour") $[1,2]$.

When a new cancer diagnosis occurs, staging examinations are performed to assess the extent of disease [3]. In this clinical scenario, it is not uncommon for the radiologist who is performing a computed tomography (CT) study to find suspicious lesions, which are often superficially classified as metastases but instead could be MPMs [4,5].

In particular, discovery of another incidental primary malignancy changes significantly the patient management. For example, in some cases the second primary tumour may require treatment more urgently than the first malignancy, for which therapy would then be deferred $[5,6]$.

Thus, radiologists should be able to detect the multiple primary tumours revealed by CT, and should not automatically consider any additional CT finding as due to a metastasis, especially for those lesions located outside the frequent sites of tumour metastasis $[7,8]$.

Aim of our retrospective, single-cancer-centre study was to assess the incidence of second, occult primary tumours in a large population undergoing staging CT, and to determine the type and extent of these tumours. This was done to identify any possible correlation, establishing which tumours are more frequently combined with a second tumour, and which second tumours are more commonly present.

We believe that the radiologist's knowledge of the prevalence and pattern of occurrence of MPMs represents added diagnostic value.

\section{Material and methods}

We reviewed the clinical charts of adult patients with a newly diagnosed cancer evaluated from January 1, 2014 to December 31, 2019 in a single cancer institute of the metropolitan area of Naples, Southern Italy.

According to Warren and Gate's criteria [9], a second tumour was defined as a previously unknown malignancy of a different histological type detected in the same organ of the first primary cancer or in another organ. All of the primary tumours in MPMs patients occurring simultaneously or within six months were classified as synchronous.

Inclusion criteria were: availability of good-quality staging CT studies and availability of a definitive diagnosis for all tumours present in the same patient. Patients with central nervous system (CNS) tumours were not included in our study, having been assessed in the Neuroradiology Unit by other colleagues. Also, only patients older than 18 years were included because young subjects are not admitted to our institution.

The enrolled patients harboured MPMs in different systems, including digestive tumours, neck cancers, lung cancers, urinary tumours, reproductive tumours, breast tumours, haematological malignancies, and endocrine tumours. The pathological types and clinical stages of malignancies were stratified separately according to the $3^{\text {rd }}$ Edition of the International Classification of Diseases for Oncology (ICD-O) [9] and the $7^{\text {th }}$ Edition of the American Joint Committee on Cancer (AJCC) [10].

CT was used to investigate all morphologic and dynamic features of neck, chest, abdomen, and pelvis primary tumours and their patterns of local, nodal, and haematogenous tumour spread. The identification of nodal involvement on CT was based on nodal size, typically using a minimum cut-off of $1 \mathrm{~cm}$ in short-axis diameter, although this approach may have led to both under- and over-staging of metastatic nodal involvement.

The standard of reference for confirming the presence or absence of malignancy was either histopathology (biopsy or surgery) or clinical-radiological follow-up for at least six months, based on Response Criteria In Solid Tumours (RECIST) [11]. Tumour progression in the follow-up CT scan or decrease in lesion size during ongoing systemic therapy were considered positive for the presence of malignancy. Lack of change or decrease in size of a lesion without ongoing systemic therapy over at least a six-month interval was considered negative for malignancy.

CT scanning of the neck, chest, abdomen, and pelvis was performed using a 64-slice multidetector CT (MDCT) scanner (LightSpeed VCT, GE Healthcare). All patients received a bolus injection $(2.5 \mathrm{cc} / \mathrm{s})$ of non-ionic iodinated contrast media (Iopamiro $370 \mathrm{mgI} / \mathrm{ml} \mathrm{Bracco}$ Imaging, Milan, Italy) followed by $200 \mathrm{cc}$ of saline solution with a dual-head injector (Stellant Injection System, Medrad Inc., United States). The administered amount of CT contrast agents was adjusted according to age and weight. CT scanning was done using three volumes, with the first volume including the chest, abdomen, and pelvis in the unenhanced phase, the second volume including the neck and chest in the arterial phase, and the third volume including the chest, abdomen, and pelvis in the portal venous phase. Specifically, arterial and portal venous acquisitions were performed with fix scan delays of 35 and $80 \mathrm{~s}$ after intravenous bolus injection of iodinated contrast media.

Technical scan parameters were the following: detector configuration $=1 \times 32 \mathrm{~mm}$; table feed $36 \mathrm{~mm} / \mathrm{s}$; rotation time $=0.75 \mathrm{~s}$; helical pitch $=27$; section thickness $=5 \mathrm{~mm}$; $120 \mathrm{kVp}$; automated tube current modulation. From the raw data of the acquisition, $3 \mathrm{~mm}$ thick transverse sections were reconstructed with $1.5 \mathrm{~mm}$ increments. Tube voltage and tube setting were $120 \mathrm{kV}$ and $130 \mathrm{mAs}$ for the neck and chest region and $120 \mathrm{kV}$ and $200 \mathrm{mAs}$ for the abdominal and pelvic region with application of automatic tube current modulation. The computed tomography dose index volume $\left(\mathrm{CTDI}_{\mathrm{vol}}\right)$ ranged between 3.0 and $5.0 \mathrm{mGy}$. 
Categorical variables are presented as the number of patients and percentages. Fisher's exact test was used for statistical comparisons of the two groups (with and without second tumour). It was used also to calculate the relative risk for each risk factor considered, including patient age, sex, and ethnic correlation. $P$-values less than 0.05 were considered statistically significant. All statistical analyses were performed using MedCalc for Microsoft Windows (version 13.1.2.0, MedCalc).

\section{Results}

Overall, 1247 subjects (18-88 years old, mean 50.1 years) were enrolled. These included 653 males (52.4\%) and 594 females (47.6\%). There were 1202 patients without CT evidence of a second tumour (prevalence $96.4 \%$, including 625 males and 577 females - mean age 50.6 years) and 45 patients with CT detection of a second tumour (prevalence 3.6\%, including 28 males and 17 females mean age 64.1 years) (Table 1). Among MPM patients, the relative risk increased with patient age and male sex, while no ethnic correlation was found.

The second tumour could be classified as non-advanced in 16/45 cases (35.6\%) and as advanced in 29/45 cases $(64.4 \%)$. In one case only the second tumour was in the same organ as the first one (hepatocellular carcinoma [HCC] plus cholangiocellular carcinoma). In all the remaining cases, the second tumour was in a different organ.

Among the patients with a second tumour, the most frequent first primary tumours were melanoma (eight patients, 17.8\%) (Figure 1), lymphoma (seven patients, $15.6 \%$ ) (Figures 2 and 3), and prostate carcinoma (seven patients, 15.6\%) (Table 2 and Figure 4).

The most frequent incidentally detected second tumours were hepatocellular carcinoma (nine patients, $20 \%$ of 45 incidental tumours) (Figure 5), renal carcinoma (eight patients, 17.8\%) (Figure 6), lung carcinoma (seven patients, 15.6\%) (Figure 7), and bladder carcinoma (four patients, $8.9 \%$ ) (Figure 8 ). One patient had three primary tumours synchronously. The distribution of second tumours according to tumour type is shown in the histogram in Figure 9. In two patients, a synchronous occurrence of primary tumours in which one of them showed a multifocal growth pattern was found. Specifically, the multifocal tumours were one HCC (Figure 1) and one bladder carcinoma (Figure 10).

\section{Discussion}

The first report about MPMs was in 1889 by Billroth, which described a patient with a spinocellular epithelioma of the right ear and a gastric carcinoma [12]. Until 1932, when Warren and Gates classified 1259 such patients from literature reports and post-mortem examinations, only a few such cases had been recognised [13]. Since that
Table 1. Demographic characteristics of the cancer population enrolled

\begin{tabular}{|l|c|c|c|} 
Parameter & $\begin{array}{c}\text { Patients without } \\
\text { a second tumour, } \\
n=1202\end{array}$ & $\begin{array}{c}\text { Patients with } \\
\text { a second tumour, } \\
n=45\end{array}$ & $\begin{array}{c}\text { Total } \\
\text { population, } \\
N=1247\end{array}$ \\
\hline Mean age & 61 years & 74 years & 62 years \\
\hline Males & 625 & 28 & 653 \\
\hline Females & 577 & 17 & 594 \\
\hline
\end{tabular}

time, numerous series and case reports in the literature have cited similar occurrences involving a single organ or multiple organ systems $[1,2]$.

In recent years, the incidence of MPMs is increasing and is expected to continue to do so. This increase has been ascribed to several factors, such as better imaging detection of many forms of cancer, longer mean lifetime, mostly in Western populations, treatments at initial tumour staging, quality of oncologic follow-up, and better prognosis of many neoplasms [14-16]. According to the literature, $1.2-3.5 \%$ of cancer patients are unexpectedly affected by a new synchronous neoplasia detected during a diagnostic or therapeutic phase [17]. Overall, the risk of
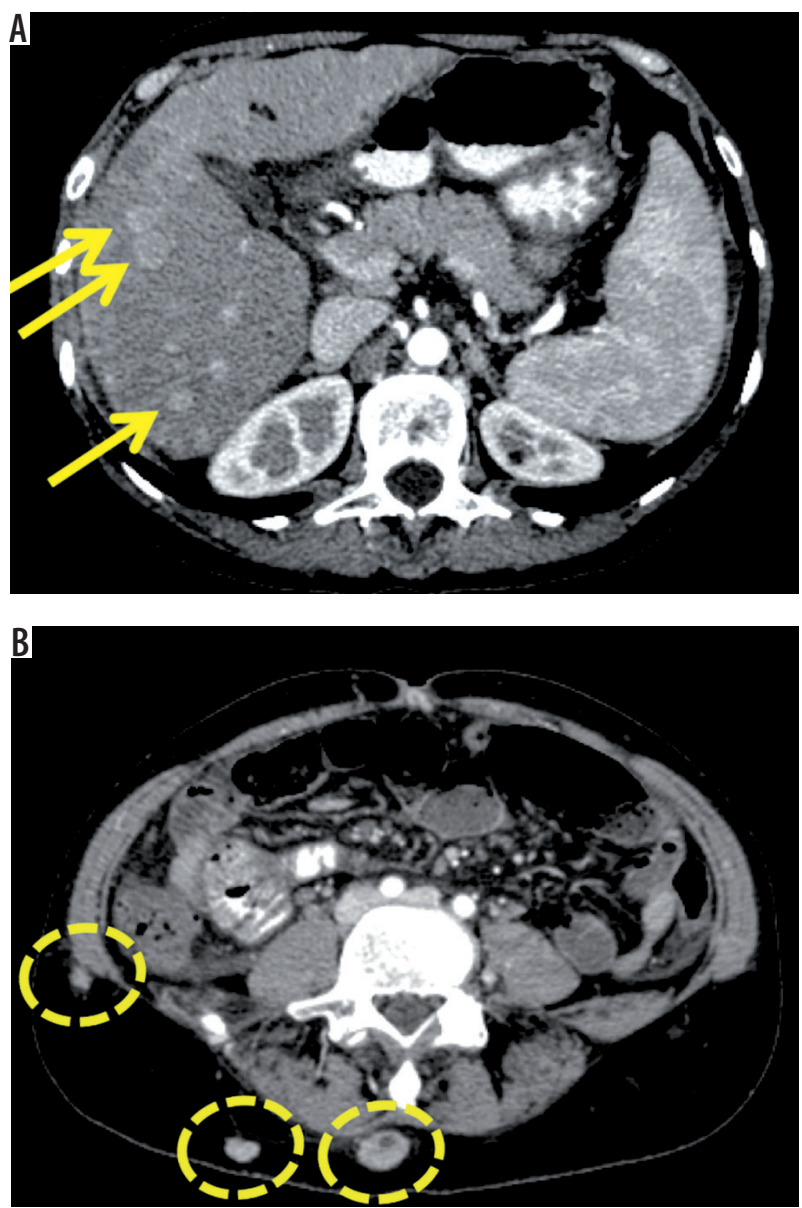

Figure 1. Axial contrast-enhanced computed tomography (CT) scans. Incidental detection of multifocal hepatocellular carcinoma (HCC) (A) (yellow arrows point to $\mathrm{HCC}$ nodules) in a 58-year-old female patient undergoing CT staging for metastatic cutaneous melanoma (B) (dashed circles indicate melanoma metastases) 

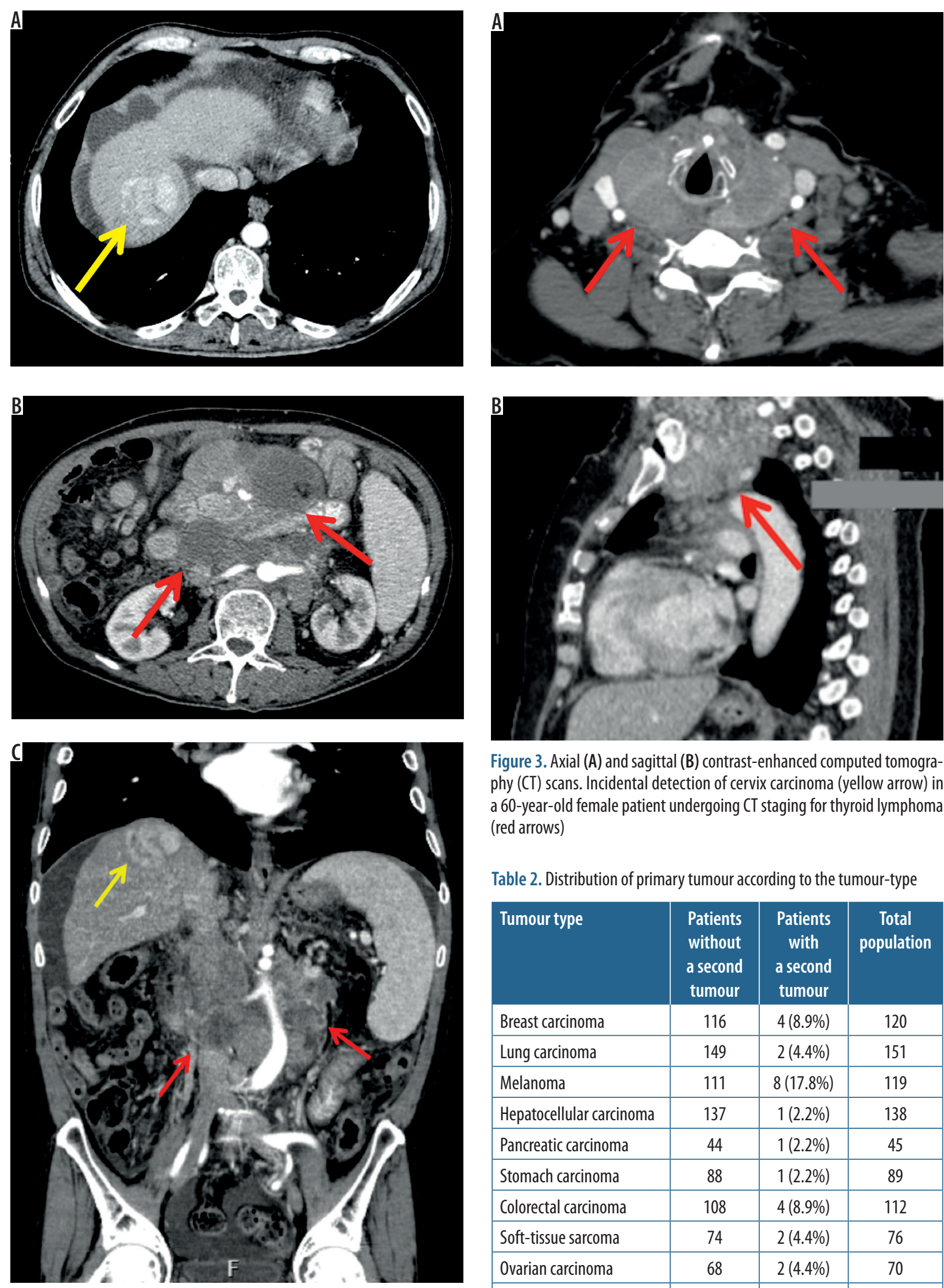

Figure 3. Axial (A) and sagittal (B) contrast-enhanced computed tomography (CT) scans. Incidental detection of cervix carcinoma (yellow arrow) in a 60 -year-old female patient undergoing CT staging for thyroid lymphoma (red arrows)

Table 2. Distribution of primary tumour according to the tumour-type

\begin{tabular}{|l|c|c|c|}
\hline Tumour type & $\begin{array}{c}\text { Patients } \\
\text { without } \\
\text { a second } \\
\text { tumour }\end{array}$ & $\begin{array}{c}\text { Patients } \\
\text { with } \\
\text { a second } \\
\text { tumour }\end{array}$ & $\begin{array}{c}\text { Total } \\
\text { population }\end{array}$ \\
\hline Breast carcinoma & 116 & $4(8.9 \%)$ & 120 \\
\hline Lung carcinoma & 149 & $2(4.4 \%)$ & 151 \\
\hline Melanoma & 111 & $8(17.8 \%)$ & 119 \\
\hline Hepatocellular carcinoma & 137 & $1(2.2 \%)$ & 138 \\
\hline Pancreatic carcinoma & 44 & $1(2.2 \%)$ & 45 \\
\hline Stomach carcinoma & 88 & $1(2.2 \%)$ & 89 \\
\hline Colorectal carcinoma & 108 & $4(8.9 \%)$ & 112 \\
\hline Soft-tissue sarcoma & 74 & $2(4.4 \%)$ & 76 \\
\hline Ovarian carcinoma & 68 & $2(4.4 \%)$ & 70 \\
\hline Uterine carcinoma & 67 & $2(4.4 \%)$ & 69 \\
\hline Prostate carcinoma & 81 & $7(15.6 \%)$ & 88 \\
\hline Lymphoma & 83 & $7(15.6 \%)$ & 90 \\
\hline Other primary tumours & 76 & $4(8.9 \%)$ & 80 \\
\hline
\end{tabular}


developing a second primary malignancy varies in different cancer sites and is reported to range from $1 \%$ (primary liver malignancy) to $16 \%$ (primary bladder cancer) [18].

To date, there is no universal diagnostic technique with overall oncologic accuracy, neither are there diagnostic guidelines to detect synchronous MPMs [6]. The choice between CT, magnetic resonance imaging (MRI), positron emission tomography (PET), and even ultrasound (US) depends on the tumour type or body region $[19,20]$.

The most widespread and recommended method for cancer staging is contrast-enhanced multidetector CT (MDCT) because it allows total-body evaluation within an extremely short time frame and, through volumetric acquisition, provides a multiplanar reconstruction of the tissues and organs of interest [21,22].

Both the primary tumour and associated tumours in adjacent and/or distant organs can be detected [23]. However, despite the interesting perspective that is achieved by high-quality multiplanar reconstruction MDCT imaging and its utility in the correct assessment of tumour extension, CT sensitivity in discovering further tumours is relatively low (85-90\%), especially in the case of lesions not distinguishable from benign abnormality (e.g. cutaneous melanoma, small thyroid tumour, or prostate carcinoma) [24].

To maximise the efficiency of contrast-enhanced MDCT to be a real "one-stop-shop" oncology examination,
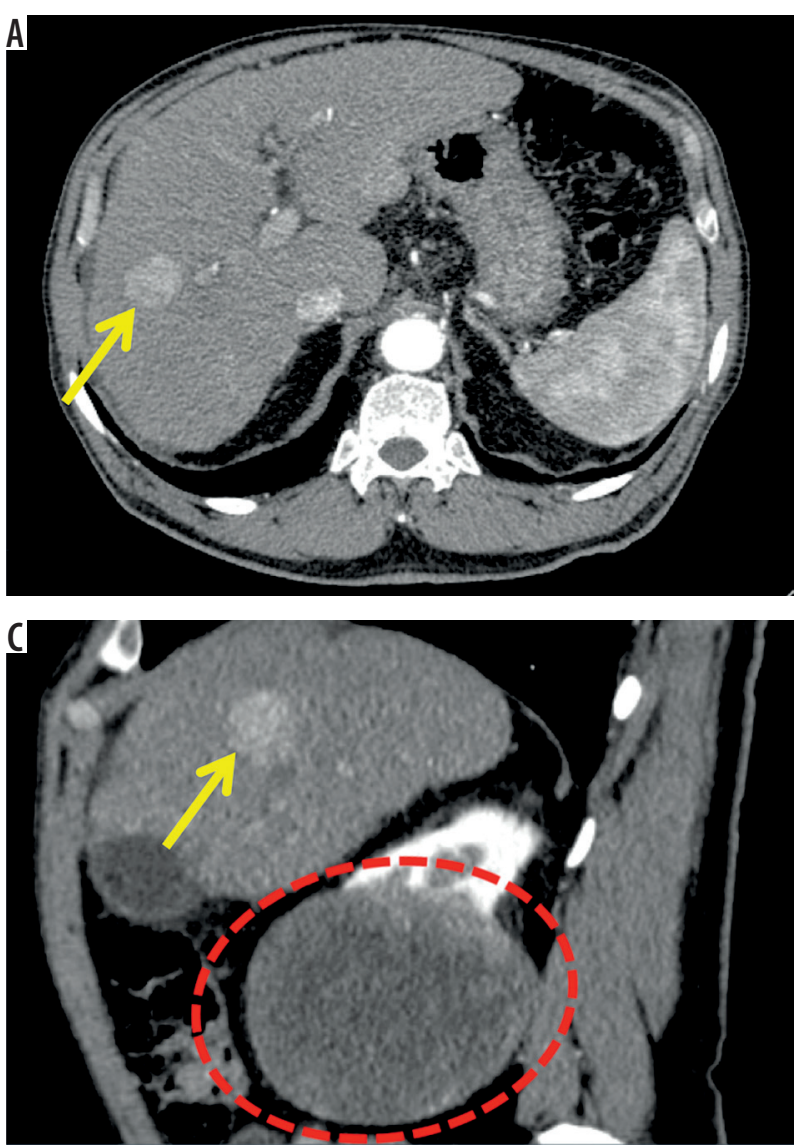

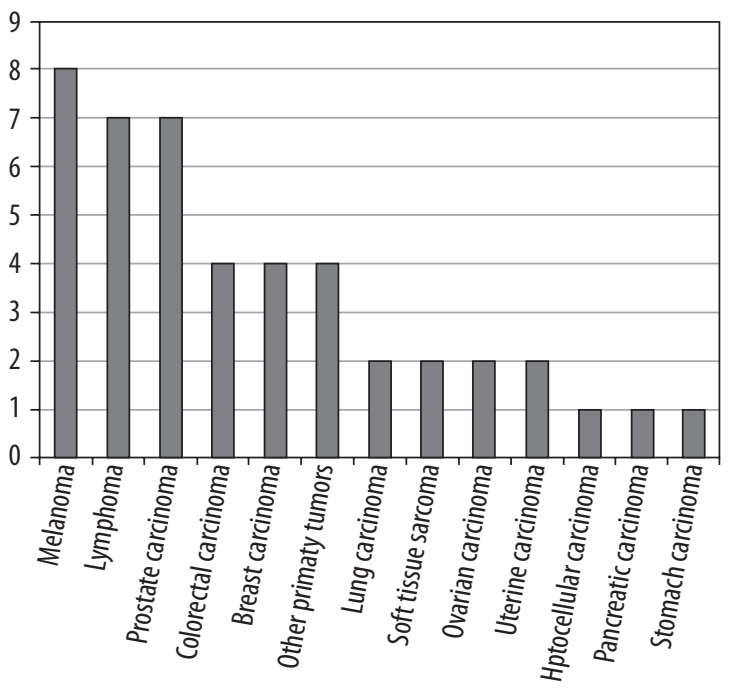

Figure 4. Distribution of first tumour according to the tumour-type

a controversial issue refers to the optimal scanning proto$\mathrm{col}$. The question is which protocol is needed to address as many specific diagnostic problems as possible. Currently there are no universal diagnostic CT protocols [25].

In order to better detect both metastatic disease and MPMs, administration of intravenous contrast material with a multiphasic approach is essential $[25,26]$. The routine use of intravenous media, unless contraindicated in some patients, helps to delineate sites of disease, especially within solid organs. In our institution, all patients

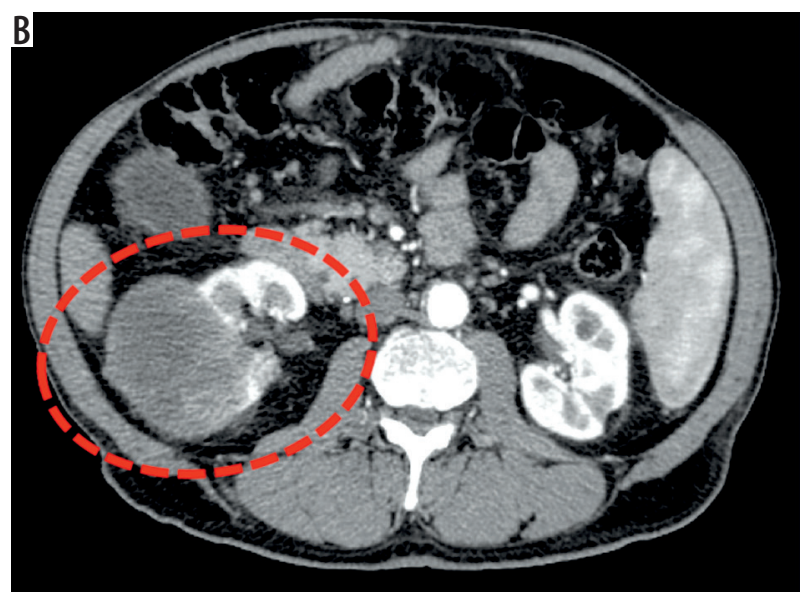

Figure 5. Axial (A, B) and sagittal (C) contrast-enhanced computed tomography (CT) scans. Incidental detection of hepatocellular carcinoma (A) (yellow arrow) in a 68-year-old male patient undergoing (T staging for right kidney clear-cell carcinoma (B) (dashed circle). C) Both tumours are shown on sagittal CT scan 

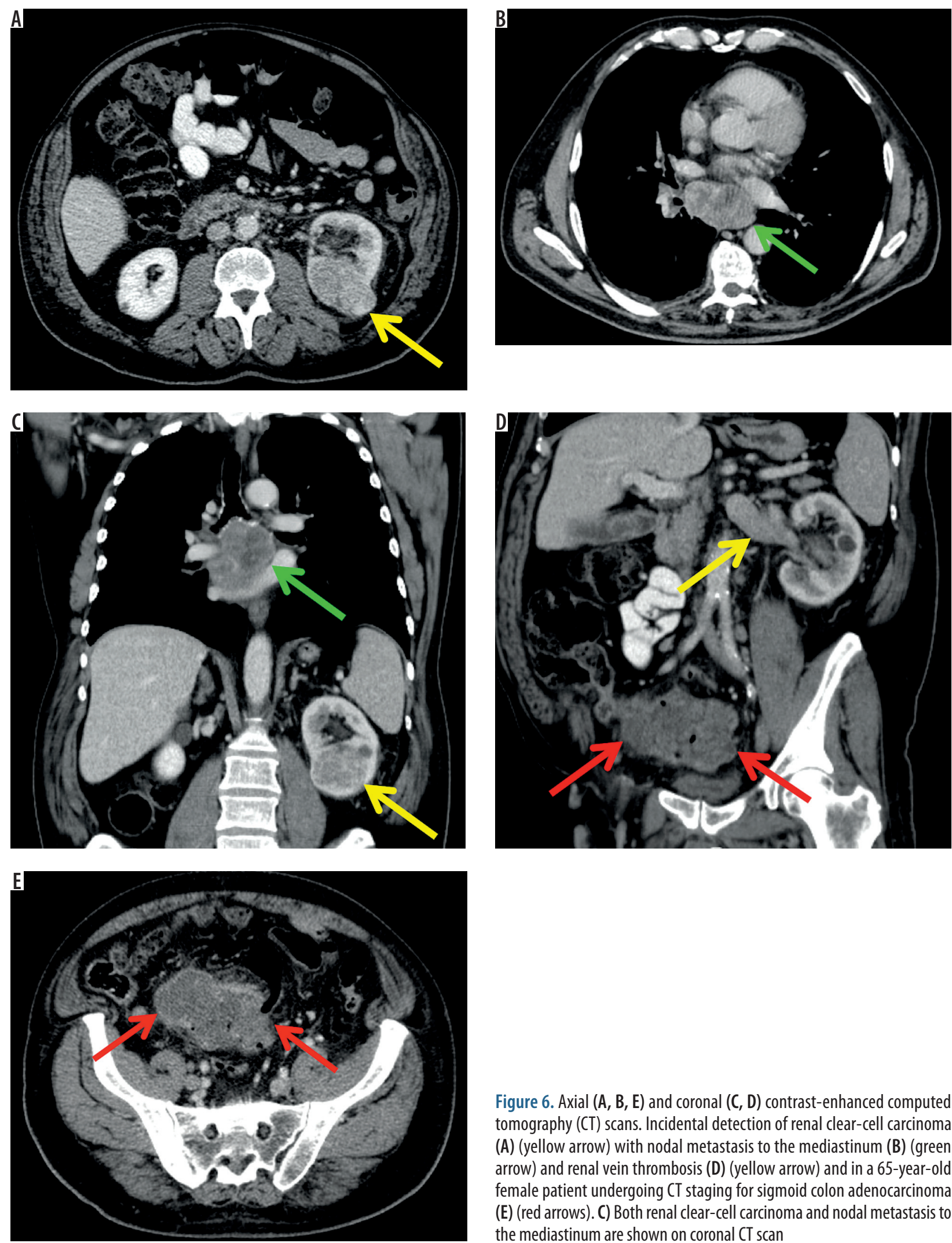

Figure 6. Axial (A, B, E) and coronal (C, D) contrast-enhanced computed tomography (CT) scans. Incidental detection of renal clear-cell carcinoma (A) (yellow arrow) with nodal metastasis to the mediastinum (B) (green arrow) and renal vein thrombosis (D) (yellow arrow) and in a 65 -year-old female patient undergoing (T staging for sigmoid colon adenocarcinoma (E) (red arrows). C) Both renal clear-cell carcinoma and nodal metastasis to the mediastinum are shown on coronal CT scan

underwent a contrast-enhanced CT with a three-phase approach including unenhanced, arterial, and portal venous acquisition. Similarly, scanning volumes must be appropriate to ensure a "diagnostic CT" [25]. In patients with tumours of the neck-chest-abdomen-pelvis, we normally limited our total-body scan to an examination of the base of the skull, neck, chest, and abdomen down to the upper thighs. In patients with malignant melanoma or peripheral sarcoma, instead, the upper extremities and lower extremities were included in the scan, but at the cost of an increase in examination time and dose to the patient. 


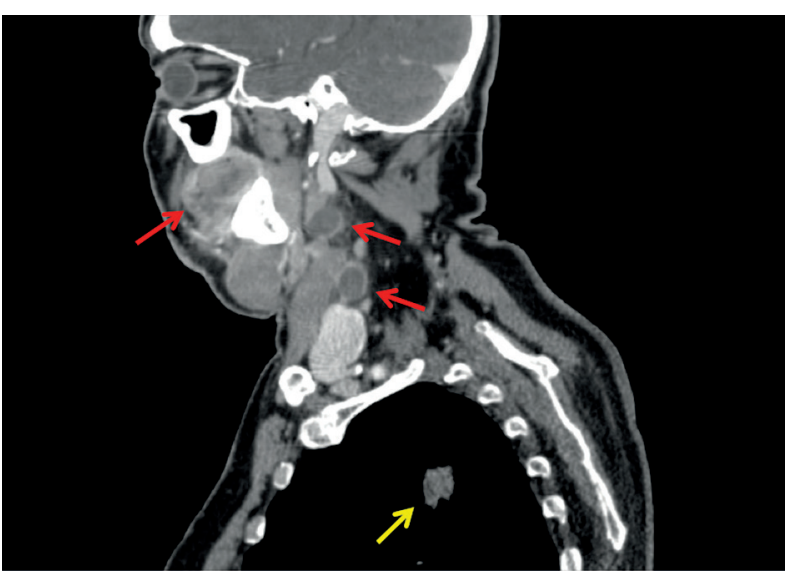

Figure 7. Sagittal contrast-enhanced computed tomography (CT) scan. Incidental detection of lung adenocarcinoma (yellow arrow) in a 47-year-old female patient undergoing $\mathrm{CT}$ staging for cheek cutaneous melanoma with cervical lymph node metastasis (red arrows)

Minimising the radiation dose delivered to patients while preserving the diagnostic accuracy of the CT examination should be a radiologist's duty in the current era of MDCT technology [27]. Indeed, radiologists should always attempt to obtain a diagnostic benefit-torisk ratio as high as reasonably achievable (AHARA), and such a radiation consciousness should primarily involve patients under 30 years old because the lifetime risk of radiation-induced cancer is greater the younger the patient. Although several dose-reduction strategies can be successfully applied in contrast-enhanced MDCT, such as the use of iterative reconstruction algorithms combined with either low tube voltage or low tube current protocols, these technologies are still not widely available in clinical practice. Thus, dose reduction in MDCT should primarily result from the optimisation of acquisition protocols, because unindicated acquisition phases or inappropriately wide scan volumes in contrast-enhanced MDCT are a major source of unjustified radiation exposure $[27,28]$.

Techniques that non-invasively yield in vivo information on cellular metabolism, such as PET, can be used in cancer assessment to detect unexpected tumour sites based on the increased uptake of radioactive tracer [29]. In clinical practice, the most commonly used radiotracer is ${ }^{18} \mathrm{~F}$-labelled fluorodeoxyglucose (FDG). Cancer lesions accumulate higher concentrations of radiotracer than normal tissue and are therefore recognisable as areas of increased radioactive intensity, which can be quantified and followed over time [30].

PET-CT scanners combine the morpho-functional images of PET with the anatomical images provided by CT. These devices offer the advantage of an accurate correspondence between areas of physiological and pathological radioactive tracer collection and anatomical landmarks, an essential feature in the correct interpretation of PET images, and thus reduce the number of false positives. Overall, the reported sensitivity and specificity of
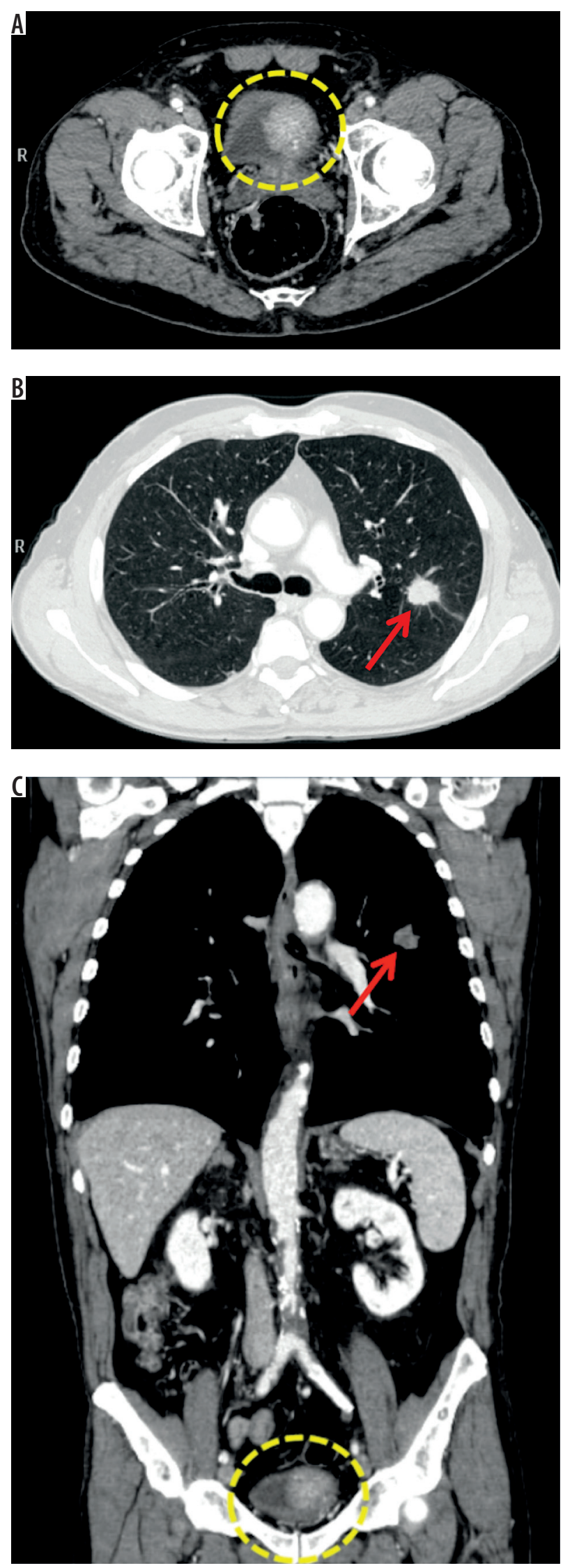

Figure 8. Axial (A, B) and coronal (C) contrast-enhanced computed tomography (CT) scans. Incidental detection of bladder carcinoma (A) (dashed circle) in a 76-year-old male patient undergoing (T staging for epidermoid lung carcinoma (B) (red arrow). C) Both tumours are shown on coronal CT scan 


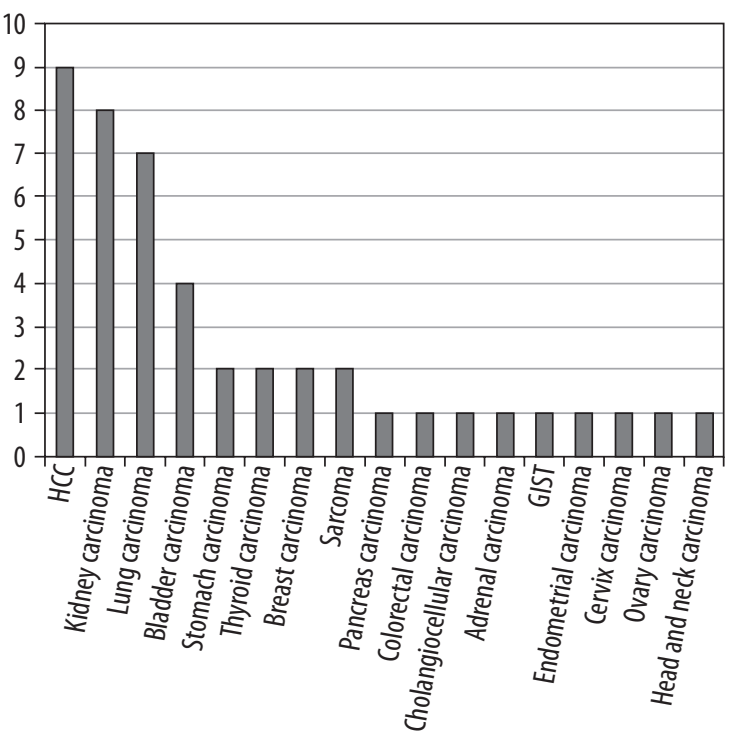

Figure 9. Distribution of second tumour according to the tumour-type

PET-CT in the detection of MPMs has been demonstrated to be, respectively, $91 \%$ and $69 \%$ [31-33].

Among intrinsic limitation of 18FDG-techniques, there is no qualitative distinction between inflammatory and neoplastic tissue, and it is often difficult to assess organs that tend to concentrate large amounts of FDG (e.g. thyroid, liver, bladder). Also, several kinds of tumours are not FDG-amenable, such as renal cell carcinoma, prostate cancer, and gastric lesions, particularly small ones, in which the tracer concentration is limited, such as early gastric cancer, deep liver lesions, and low-grade soft-tissue malignancies. In the absence of pronounced morphological changes, this may lead to false-negatives in early-stage disease. Furthermore, the high cost of PET-CT, its limited availability restricted to a few specialised centres, and the guidelines limiting the amount of radiation allowed for each first-stage diagnostic session restrict its use [6].

For these reasons, baseline FDG-PET is not extensively performed in clinical practice, and the majority of these examinations were acquired after CT studies. Thus, CT remains the most commonly used imaging modality for staging newly diagnosed tumours, and in some cases it is the only imaging test needed prior to surgical management. This aspect further confirms the usefulness of our research.

Our study had some limitations. First, a selection bias. Although we used a population-based surveillance system, we studied only data from a single hospital. In addition, the patients enrolled were selected from a population of patients entering in our cancer institute, which is a regional reference centre in the diagnosis and treatment of melanomas and lymphomas. Therefore, it was obvious that melanoma and lymphoma patients had a high prevalence of MPMs. Second, only patients with newly diagnosed synchronous MPMs were included, and the results of this study may not apply to other, larger MPMs patient populations. Third, our retrospective study includes data for an observation period of only five years. In our
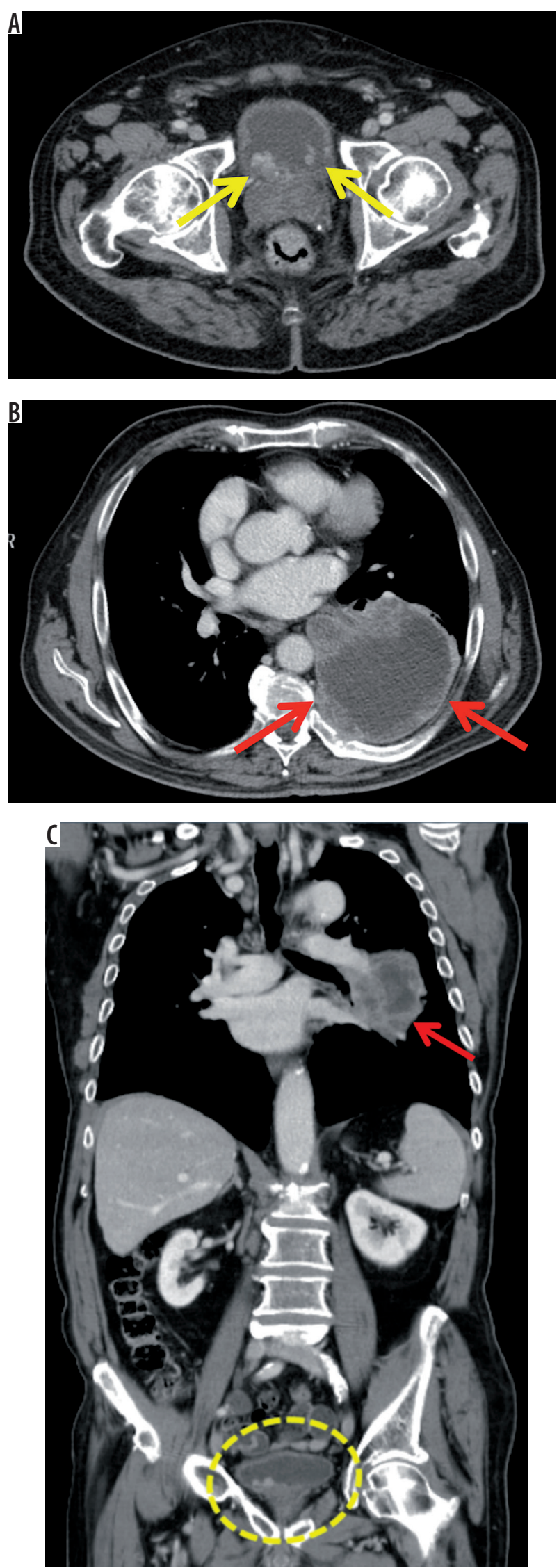

Figure 10. Axial (A, B) and coronal (C) contrast-enhanced computed tomography (CT) scans. Incidental detection of multifocal bladder carcinoma (A) (yellow arrows and dashed circle) in a 70-year-old male patient undergoing (T staging for lung adenocarcinoma (B) (red arrows). C) Both tumours are shown on coronal CT scan 
opinion, a more sophisticated analysis of MPMs requires a larger, well-defined population under observation for $\geq 10$ years. Fourth, patients with CNS tumours were not included in our study, having been assessed in the Neuroradiology Unit by other colleagues. Similarly, only patients older than 18 years were included in the study because young subjects are not admitted to our institution. And fifth, patients experience of staging CT was not compared to that of FDG-PET or FDG-PET/CT.

\section{Conclusions}

Our study deals with multiple primary tumours coexisting in the same organ or in different organs in the same patient and detected synchronously during CT staging.
Because the clinical implications and therapeutic strategies vary significantly according to a correct diagnosis, radiologists should be aware of this occurrence.

Thus, when planning the CT acquisition, radiologists should evaluate all the possibilities of using a multiphasic CT approach in order to improve the diagnostic accuracy of the examination, but they should try to avoid unindicated acquisition phases or inappropriately wide scan volumes as a source of unjustified radiation exposure.

Additionally, when reviewing the CT images, they should not automatically consider any new lesion as a metastasis. Particularly in the case of an uncommon site of metastatic lesion, a second primary tumour should be considered in the differential diagnosis.

\section{References}

1. Maurea S, Corvino A, Imbriaco M, et al. Simultaneous non-functioning neuroendocrine carcinoma of the pancreas and extrahepatic cholangiocarcinoma. A case of early diagnosis and favorable post-surgical outcome. JOP 2011; 12(3): 255-8.

2. Corvino A, Corvino F, Radice L, et al. Synchronous mucinous colonic adenocarcinoma and multiple small intestinal adenocarcinomas: report of a case and review of literature. Clin Imaging 2015; 39(3): 538-42. doi: 10.1016/j.clinimag.2014.12.019. Epub 2015 Jan 7.

3. Fritz A, Percy C, Jack A, editors. International Classification of Diseases for Oncology, third edition. Geneva, World Health Organization, 2000.

4. Demandante CG, Troyer DA, Miles TP. Multiple primary malignant neoplasms: case report and a comprehensive review of the literature. Am J Clin Oncol 2003; 26(1): 79-83.

5. Vogt A, Schmid S, Heinimann K, et al. Multiple primary tumours: challenges and approaches, a review. ESMO Open 2017; 2(2): e000172. doi: 10.1136/esmoopen-2017-000172. eCollection 2017.

6. Coyte A, Morrison DS, McLoone P. Second primary cancer risk the impact of applying different definitions of multiple primaries: results from a retrospective population-based cancer registry study. BMC Cancer 2014; 14: 272. doi: 10.1186/1471-2407-14-272.

7. Tammaro V, Spiezia S, D’Angelo S, et al. Diagnostic Imaging Techniques for Synchronous Multiple Tumors. In: Renda A (ed.). Updates in Surgery. Multiple Primary Malignancies. Springer-Verlag 2009; p. 231-244.

8. Dijkman BG, Schuurbiers OC, Vriens D, et al. The role of (18) F-FDG PET in the differentiation between lung metastases and synchronous second primary lung tumours. Eur J Nucl Med Mol Imaging 2010; 37(11): 2037-47. doi: 10.1007/s00259-010-1505-2. Epub 2010 Jun 10.

9. Warren S, Gates O. Multiple primary malignant tumors: a survey of the literature and statistical study. Am J Cancer 1932; 16: 1358-414.

10. Stephen BE, David RB, Carolyn CC, editors. AJCC Cancer Staging Manual. 7th ed. Springer, New York, USA 2010.

11. Feinberg BA, Bharmal M, Klink AJ, et al. Using Response Evaluation Criteria in Solid Tumors in real-world evidence cancer research.
Future Oncol 2018; 14(27): 2841-2848. doi: 10.2217/fon-2018-0317. Epub 2018 May 31.

12. Renda A, Carlomagno N. Nosography. In: Renda A (ed.). Updates in Surgery. Multiple Primary Malignancies 2009; p. 231-244.

13. Testori A, Cioffi U, De Simone M, et al. Multiple primary synchronous malignant tumors. BMC Res Notes 2015; 8: 730. doi: 10.1186/ s13104-015-1724-5.

14. Amer MH. Multiple neoplasms, single primaries, and patient survival. Cancer Manag Res 2014; 6: 119-34. doi: 10.2147/CMAR. S57378. eCollection 2014.

15. Luciani A, Balducci L. Multiple primary malignancies. Semin Oncol 2004; 31(2): 264-73

16. Skelton WP 4th, Ali A, Skelton MN, et al. Analysis of Overall Survival in Patients With Multiple Primary Malignancies: A Single-center Experience. Cureus 2019; 11(4): e4552. doi: 10.7759/ cureus. 4552 .

17. Artac M, Bozcuk H, Ozdogan M, et al. Different clinical features of primary and secondary tumors in patients with multiple malignancies. Tumori 2005; 91(4): 317-20.

18. Hayat MJ, Howlader N, Reichman ME, et al. Cancer statistics, trends, and multiple primary cancer analyses from the Surveillance, Epidemiology, and End Results (SEER) Program. Oncologist 2007; 12(1): 20-37.

19. Lee JC, Teles MS. Prevalence of additional primary malignancies detected incidentally on PET/CT. Radiol Bras 2019; 52(5): 348. doi: 10.1590/0100-3984.2019.0097.

20. Corvino A, Rosa D, Sbordone C, et al. Diastasis of rectus abdominis muscles: patterns of anatomical variation as demonstrated by ultrasound. Pol J Radiol 2019; 84: e542-e548. doi: 10.5114/ pjr.2019.91303. eCollection 2019.

21. Ros PR, Ji H. Special focus session: multisection (multidetector) CT: applications in the abdomen. Radiographics 2002; 22(3): 697-700.

22. Kalra MK, Maher MM, D'Souza R, et al. Multidetector computed tomography technology: current status and emerging developments. J Comput Assist Tomogr 2004; 28 Suppl 1: S2-6.

23. Tibana TK, Santos RFT, Arão Filho A, et al. Detection of additional primary malignancies: the role of CT and PET/CT combined with 
multiple percutaneous biopsy. Radiol Bras 2019; 52(3): 166-171. doi: 10.1590/0100-3984.2018.0024.

24. Ueda T, Mori K, Minami M, et al. Trends in oncological CT imaging: clinical application of multidetector-row CT and 3D-CT imaging. Int J Clin Oncol 2006; 11(4): 268-77.

25. Ertekin E, Amasyalı AS, Erol B, et al. Role of Contrast Enhancement and Corrected Attenuation Values of Renal Tumors in Predicting Renal Cell Carcinoma (RCC) Subtypes: Protocol for a Triphasic Multi-Slice Computed Tomography (CT) Procedure. Pol J Radiol 2017; 82: 384-391. doi: 10.12659/PJR.901957. eCollection 2017.

26. Pfannenberg AC, Aschoff P, Brechtel K, et al. Value of contrast-enhanced multiphase CT in combined PET/CT protocols for oncological imaging. Br J Radiol 2007; 80(954): 437-45. Epub 2007 Feb 28.

27. Thrall JH. Radiation exposure in CT scanning and risk: where are we? Radiology 2012; 264: 325-8. doi: https://doi.org/10.1148/ radiol.12121137.

28. Brenner DJ, Hall EJ. Computed tomography: an increasing source of radiation exposure. N Engl J Med 2007; 357: 2277-84. doi: https:// doi.org/10.1056/NEJMra072149
29. Phelps ME. Positron emission tomography provides molecular imaging of biological processes. Proc Natl Acad Sci U S A 2000; 97(16): 9226-33.

30. Veit P, Kühle C, Beyer T, Kuehl H, et al. Whole body positron emission tomography/computed tomography (PET/CT) tumour staging with integrated PET/CT colonography: technical feasibility and first experiences in patients with colorectal cancer. Gut 2006; 55(1): 68-73.

31. Townsend DW, Beyer T. A combined PET/CT scanner: the path to true image fusion. Br J Radiol 2002; 75 Spec No: S24-30.

32. Antoch G, Freudenberg LS, Beyer T, et al. To enhance or not to enhance? 18F-FDG and CT contrast agents in dual-modality 18F-FDG PET/CT. J Nucl Med 2004; 45 Suppl 1: 56S-65S.

33. Choi JY, Lee KS, Kwon OJ, et al. Improved detection of second primary cancer using integrated $[18 \mathrm{~F}]$ fluorodeoxyglucose positron emission tomography and computed tomography for initial tumor staging. J Clin Oncol 2005; 23(30): 7654-9. 